\title{
On the Boundedness Property of the Inertia Matrix and Skew-Symmetric Property of the Coriolis Matrix for Vehicle-Manipulator Systems
}

\author{
Pål Johan From \\ Department Mathematical Sciences and Technology, \\ Norwegian University of Life Sciences, Ås, Norway \\ Email: pafr@umb.no
}

\author{
Ingrid Schjølberg \\ Applied Cybernetics, \\ SINTEF, \\ Trondheim, Norway
}

\author{
Jan Tommy Gravdahl, Kristin Ytterstad Pettersen, Thor I. Fossen \\ Department of Engineering Cybernetics, \\ Norwegian University of Science and Technology, Trondheim, Norway
}

This paper addresses the boundedness property of the inertia matrix and the skew-symmetric property of the Coriolis matrix for vehicle-manipulator systems. These properties are widely used in control theory and Lyapunov-based stability proofs and thus important to identify. The skew-symmetric property does not depend on the system at hand, but on the parameterisation of the Coriolis matrix, which is not unique. It is the authors' experience that many researchers take this assumption for granted without taking into account that several parameterisations exist. In fact, most researchers refer to references that do not show this property for vehiclemanipulator systems, but for other systems such as single rigid bodies or fixed-base manipulators. As a result, the otherwise rigorous stability proofs fall apart. In this paper we list some relevant references and give the correct proofs for some commonly used parameterisations for future reference.

Depending on the choice of state variables, the boundedness of the inertia matrix will not necessarily hold. We show that deriving the dynamics in terms of quasi-velocities leads to an inertia matrix that is bounded in its variables. To the authors' best knowledge we derive for the first time the dynamic equations of vehicle-manipulator systems with non-Euclidean joints for which both properties are true.

\section{Introduction}

This paper is motivated by a general concern that some frequently used properties of the inertia and Coriolis matrices for vehicle-manipulator systems are assumed true based on the proofs for other systems. We show that the proofs of these properties for fixed-base robot manipulators or single rigid bodies cannot be generalised to vehicle-manipulator systems directly. In fact, we show that the most commonly used equations for vehicle-manipulator systems do not pos- sess both the boundedness and skew-symmetric properties. There is thus a need to clarify to what extent these properties are true and find rigorous mathematical representations of these systems for use in simulations and controller design. To this end we present a reformulation of the dynamic equations for vehicle-manipulator systems for which both the boundedness and the skew-symmetric properties are true.

Lyapunov based controllers are based on several assumptions that make the controller design both more convenient and physically meaningful. Unfortunately, these properties are almost universally taken for granted. As these assumptions are not always true, the stability proofs fall apart.

The first property is concerned with the boundedness of the inertia matrix $M$, i.e. the existence of lower and upper bounds on its singular values. For a given robotic manipulator there may exist one mathematical representation for which the inertia matrix is bounded and another for which it is not. For the most common mathematical representation of vehicle-manipulator systems this property is not true.

The second problem that we are concerned with is to find a parameterisation of the Coriolis matrix $C$ so that the matrix $\dot{M}-2 C$ is skew-symmetric. Such a parameterisation is easy to find for fixed-base robots or for single rigid bodies, but not always for vehicle-manipulator systems. Particularly we find that such a parameterisation is rather hard to find, especially together with the boundedness property. The skewsymmetric property of the Coriolis matrix is in most cases assumed true without any further proof. In the authors' view, this is a strong weakness because this property depends on how we choose to represent the Coriolis matrix. It is thus not sufficient to refer to an arbitrary proof of skew-symmetry: one must refer to a proof for the specific parameterisation of the Coriolis matrix chosen. Most papers on the topic of 
vehicle-manipulator systems refer to Antonelli [1], Fossen and Fjellstad [2], Canudas de Wit et al. [3] or Schjølberg and Fossen [4] for this proof. However, none of these references actually show the proof. Also references taken from the fixed-base robotics literature such as Murray et al. [5] and Sciavicco and Siciliano [6] are commonly and wrongly used for vehicle-manipulator systems.

The proof can be found in Schjølberg [7], but only for systems where the boundedness property does not hold. We present this proof, and correct some mistakes made, so that this proof is correctly presented for future reference. For the formulation presented in Egeland and Pettersen [8] the dynamics possesses the skew-symmetric property and, based on the proof in Schjølberg and Fossen [4], we show that this property can be shown also when the dynamics are written in terms of global state variables.

\section{Properties of the dynamics}

In this section we list some important properties of dynamical systems in matrix form that play important roles in system analysis as well as controller design. Assume for now that we can write the dynamic equations of a mechanical system in the form

$$
M(q) \ddot{q}+C(q, \dot{q}) \dot{q}=\tau
$$

where $q$ is the state of the system, $M(q)$ is the inertia matrix, and $C(q, \dot{q})$ is the Coriolis matrix. The following properties can be associated with the inertia and Coriolis matrices [9]:

Property 1. (The boundedness property) The inertia matrix $M(q)$ is uniformly bounded in q, i.e. there exist constants $d_{1}$ and $d_{2}$, such that $0<d_{1} \leq\|M(q)\| \leq d_{2}<\infty, \forall q \in \mathbb{R}^{n}$ where $\|\cdot\|$ is the induced 2-norm for matrices (see [10]), i.e. a max-bound on the maximum singular value and a minbound on the minimum singular value of the matrix.

Property 2. (The skew-symmetric property) The matrix $(\dot{M}(q)-2 C(q, \dot{q}))$ is skew-symmetric.

Property 1 is true when there are no singularities present. Thus, if the Euler angles are used to represent the attitude of the vehicle, as in Fossen [11], Schjølberg [7] and Børhaug [9], this is not satisfied. The existence of the boundaries $d_{1}$ and $d_{2}$ is the basis of gain controller design and global Lyapunov stability, and is used in several manipulator control laws such as robust control $[6,10]$.

Property 2 is true for a certain parameterisation of the Coriolis matrix. Such a representation is well known for robotic manipulators on a fixed base $[5,6]$ and for vehicles with no manipulator attached [11]. This property is frequently used to cancel the non-linearities of the Coriolis matrix from Lyapunov functions.

\section{Vehicle-Manipulator Dynamics}

In this section we review some commonly used approaches for modelling vehicle-manipulator systems.

\subsection{The Model of Schjølberg [7]}

In this section we present the dynamic equations as they are normally presented in the underwater robotics literature. The details can be found in Schjølberg [7]. The dynamics can be written as

$$
\begin{aligned}
\dot{\xi} & =J(\xi) \zeta, \\
M(q) \dot{\zeta}+C(q, \zeta) \zeta & =\tau
\end{aligned}
$$

where $\xi=\left[\begin{array}{ll}\eta^{\top} & q^{\top}\end{array}\right]^{\top}$ denotes the position, $\zeta=\left[\begin{array}{ll}v^{\top} & \dot{q}^{\top}\end{array}\right]^{\top}$ the velocity, $M(q) \in \mathbb{R}^{(6+n) \times(6+n)}$ is the inertia matrix, and $C(q, \zeta) \in \mathbb{R}^{(6+n) \times(6+n)}$ is the Coriolis matrix. The velocity transformation matrix is given by

$$
J(\xi)=\left[\begin{array}{ccc}
R_{0 b}(\Theta) & 0 & 0 \\
0 & T_{\Theta}(\Theta) & 0 \\
0 & 0 & I
\end{array}\right] \in \mathbb{R}^{(6+n) \times(6+n)},
$$

where $R_{0 b}(\Theta) \in S O(3)$ is the rotation matrix and $\Theta=$ $\left[\begin{array}{lll}\phi & \theta & \psi\end{array}\right]^{\top}$ the Euler angles. $T_{\Theta}(\Theta)$ is given by (zyx-sequence)

$$
T_{\Theta}(\Theta)=\left[\begin{array}{ccc}
1 & \sin \phi \tan \theta & \cos \phi \tan \theta \\
0 & \cos \phi & -\sin \phi \\
0 & \frac{\sin \phi}{\cos \theta} & \frac{\cos \phi}{\cos \theta}
\end{array}\right] .
$$

$T_{\Theta}(\Theta)$, and thus also $J(\xi)$, are not defined for $\theta= \pm \pi / 2$.

Let $v_{0 i}^{0}$ denote the linear and angular velocity of body $i$ represented in the inertial frame, and $P_{i}(q) \in \mathbb{R}^{6 \times(6+n)}$ be the transformation matrix of link $i$, that gives the relation $v_{0 i}^{0}=$ $P_{i}(q) \zeta$. The inertia matrix of the vehicle-manipulator system can then be written as [8]

$$
M(q)=\sum_{i=b}^{n} P_{i}^{\top}(q) I_{i} P_{i}(q)
$$

where $I_{i} \in \mathbb{R}^{6 \times 6}$ denotes the constant positive-definite diagonal inertia tensor of link $i$ expressed in $\mathcal{F}_{i}$ and we thus sum from the base $b$ to the end of the chain, i.e., link $n$. We note that the inertia matrix $M(q)$ depends only on the joint variables $q$ and is independent of the position $\eta$ of the vehicle.

The Coriolis matrix is given by [7]

$$
C(q, \zeta)=\sum_{i=b}^{n} \dot{P}_{i}^{\top}(q) I_{i} P_{i}(q)-P_{i}^{\top}(q) W_{i}(\zeta) P_{i}(q)
$$

where $W_{i}(\zeta)$ is a skew-symmetric matrix [7]. We will use the framework of Egeland and Pettersen [8] to find an expression for $W_{i}(\zeta)$. This is shown in Section 3.2.

\subsection{Multibody Dynamics in Terms of Quasi-Velocities}

In this section we derive the dynamics of a robotic manipulator mounted on a free-floating base in terms of quasi- 
velocities. The approach is based on Egeland and Pettersen [8], but a few errors from this paper have been corrected and we also provide some more details in the derivation. First, write the linear and angular velocities $v_{0 i}^{0}$ of each link $i$ represented in the inertial frame $\mathcal{F}_{0}$ as

$$
v_{0 i}^{0}=\left[\begin{array}{c}
v_{0 i, v}^{0} \\
v_{0 i, \omega}^{0}
\end{array}\right]=\frac{\partial v_{0 i}^{0} \zeta}{\partial \zeta}
$$

and the dynamics can be written as [8]

$$
\sum_{i=b}^{6}\left\{\frac{\partial v_{0 i}^{0}}{\partial \zeta}\left[\frac{\mathrm{d}}{\mathrm{d} t} \frac{\partial \mathcal{K}_{i}^{\top}}{\partial v_{0 i}^{0}}+\left[\begin{array}{cc}
\hat{\mathrm{v}}_{0 i, \omega}^{0} & 0 \\
\hat{\mathrm{v}}_{0 i, v}^{0} & \hat{\mathrm{v}}_{0 i, \omega}^{0}
\end{array}\right] \frac{\partial \mathcal{K}_{i}^{\top}}{\partial v_{0 i}^{0}}\right]\right\}=\tau
$$

We now derive the dynamics in matrix form following the approach in Egeland and Pettersen [8], but in addition we show the explicit expressions for the matrices which were not shown in Egeland and Pettersen [8] and we correct an error is the expression of the Coriolis matrix. First write

$$
\frac{\mathrm{d}}{\mathrm{d} t} \frac{\partial \mathcal{K}_{i}}{\partial v_{0 i}^{0}}=\frac{\mathrm{d}}{\mathrm{d} t}\left(I_{i} v_{0 i}^{0}\right)=I_{i} \dot{\mathrm{v}}_{0 i}^{0}=I_{i}\left(\frac{\partial v_{0 i}^{0}}{\partial \zeta} \dot{\zeta}+\frac{\partial \dot{v}_{0 i}^{0}}{\partial \zeta}\right)
$$

and

$$
\begin{aligned}
{\left[\begin{array}{cc}
\hat{\mathrm{v}}_{0 i, \omega}^{0} & 0 \\
\hat{\mathrm{v}}_{0 i, v}^{0} & \hat{\mathrm{v}}_{0 i, \omega}^{0}
\end{array}\right]\left[\begin{array}{c}
\frac{\partial \mathscr{K}_{i}}{\partial v_{0 i, v}^{0}} \\
\frac{\partial \mathscr{K}_{i}}{\partial v_{0 i, \omega}^{0}}
\end{array}\right] } & =\left[\begin{array}{cc}
-\frac{\partial \mathscr{K}_{i}}{\partial v_{0 i, v}^{0}} \times v_{0 i, \omega}^{0} & 0 \\
-\frac{\partial \mathcal{K}_{i}}{\partial v_{0 i, v}^{0}} \times v_{0 i, v}^{0} & -\frac{\partial \mathcal{K}_{i}}{\partial v_{0 i, \omega}^{0}} \times v_{0 i, \omega}^{0}
\end{array}\right] \\
& =-\left[\begin{array}{cc}
0 & \frac{\partial \mathcal{K}_{i}}{\partial v_{0 i, v}^{0}} \\
\frac{\partial \mathcal{K}_{i}}{\partial v_{0 i, v}^{0}} & \frac{\partial \mathcal{K}_{i}}{\partial v_{0 i, \omega}^{0}}
\end{array}\right]\left[\begin{array}{c}
v_{0 i, v}^{0} \\
v_{0 i, \omega}^{0}
\end{array}\right]
\end{aligned}
$$

Substituting (10) and (11) into (9) we get

$$
\begin{aligned}
& \sum_{i=b}^{6}\left\{\frac{\partial v_{0 i}^{0}{ }^{\top}}{\partial \zeta}\left[\frac{\mathrm{d}}{\mathrm{d} t} \frac{\partial \mathcal{K}_{i}^{\top}}{\partial v_{0 i}^{0}}+\left[\begin{array}{cc}
\hat{\mathrm{v}}_{0 i, \omega}^{0} & 0 \\
\hat{\mathrm{v}}_{0 i, v}^{0} & \hat{\mathrm{v}}_{0 i, \omega}^{0}
\end{array}\right] \frac{\partial \mathcal{K}_{i}^{\top}}{\partial v_{0 i}^{0}}\right]\right\}=\tau \\
& \sum_{i=b}^{6}\left\{\frac{\partial v_{0 i}^{0}}{\partial \zeta} I_{i} \frac{\partial v_{0 i}^{0}}{\partial \zeta} \dot{\zeta}+\frac{\partial v_{0 i}^{0}}{\partial \zeta} I_{i} \frac{\partial \dot{v}_{0 i}^{0}}{\partial \zeta} \zeta\right. \\
& \left.-\frac{\partial v_{0 i}^{0}}{\partial \zeta}\left[\begin{array}{cc}
0 & \frac{\partial \mathscr{K}_{i}}{\partial v_{0 i, v}^{0}} \\
\frac{\partial \mathcal{K}_{i}}{\partial v_{0 i, v}^{0}} & \frac{\partial \mathscr{K}_{i}}{\partial v_{0 i, \omega}^{0}}
\end{array}\right]\left[\begin{array}{c}
v_{0 i, v}^{0} \\
v_{0 i, \omega}^{0}
\end{array}\right]\right\}=\tau \\
& \begin{aligned}
\sum_{i=b}^{6}\left[\frac{\partial v_{0 i}^{0}}{\partial \zeta} I_{i} \frac{\partial v_{0 i}^{0}}{\partial \zeta}\right] \dot{\zeta} \\
\quad+\sum_{i=b}^{6}\left[\frac{\partial v_{0 i}^{0}}{\partial \zeta} I_{i} \frac{\partial \dot{v}_{0 i}^{0}}{\partial \zeta}-\frac{\partial v_{0 i}^{0}}{\partial \zeta}\left[\frac{0}{\frac{\partial \mathcal{K}_{i}}{\partial v_{0 i, v}^{0}}} \frac{\frac{\partial \mathcal{K}_{i}}{\partial v_{0 i, v}^{0}}}{\frac{\partial \mathcal{K}_{i}}{\partial v_{0 i, \omega}^{0}}}\right] \frac{\partial v_{0 i}^{0}}{\partial \zeta}\right] \zeta=\tau
\end{aligned}
\end{aligned}
$$

where we have used the relation in (8). The inertia matrix is then given by (6) with $P_{i}(q)=\frac{\partial v_{0 i}^{0}}{\partial \zeta}$ and the Coriolis matrix is given by (7) with

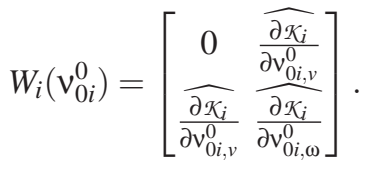

\subsection{General Multibody Dynamics}

In this section we extend the formulation from the previous section to include more general structures and also mechanisms where the position of the vehicle needs to be included in the dynamics. The approach is based on Duindam and Stramigioli [12] and From et al. [13] where the dynamics of vehicle-manipulator systems are derived and the boundedness property holds.

Using standard notation [5], we can describe the pose of each frame $\mathcal{F}_{i}$ relative to $\mathcal{F}_{0}$ as a homogeneous transformation matrix $g_{0 i} \in S E(3)$. This pose can also be described using the vector of joint coordinates $q$ as $g_{0 i}=g_{0 b} g_{b i}=$ $g_{0 b} g_{b i}(q)$. The base pose $g_{0 b}$ and the joint positions $q$ thus fully determine the configuration state of the robot. In a similar way, the spatial velocity of each link can be expressed using twists [5]:

$$
v_{0 i}^{0}=\left[\begin{array}{c}
v_{0 i, v}^{0} \\
v_{0 i, \omega}^{0}
\end{array}\right]=v_{0 b}^{0}+v_{b i}^{0}=\operatorname{Ad}_{g_{0 b}}\left(v_{0 b}^{b}+J_{i}(q) \dot{q}\right)
$$

where $v_{0 i, v}^{0}$ and $v_{0 i, \omega}^{0}$ are the linear and angular velocities, respectively, of link $i$ relative to the inertial frame, $J_{i}(q) \in \mathbb{R}^{6 \times n}$ is the geometric Jacobian of link $i$ relative to $\mathcal{F}_{b}$ and the adjoint is defined as $\operatorname{Ad}_{g}:=\left[\begin{array}{cc}R & \hat{p} R \\ 0 & R\end{array}\right] \in \mathbb{R}^{6 \times 6}$. The velocity state is thus fully determined given the twist $v_{0 b}^{b}$ of the base and the joint velocities $\dot{q}$. This illustrates how the kinematics of the system can be naturally described in terms of the (global) state variables $Q=\left\{g_{0 b}, q\right\}$ and $v=\left\{v_{0 b}^{b}, \dot{q}\right\}$.

Given a mechanism with coordinates formulated in this generalised form, we can write its kinetic energy as $\mathcal{K}(Q, v)=\frac{1}{2} v^{\top} M(Q) v$ with $M(Q)$ the inertia matrix in coordinates $Q$. The dynamics of this system then satisfies

$$
M(Q) \dot{v}+C(Q, v) v=\tau
$$

with $\tau$ the vector of gravitational forces, friction, and other external forces (collocated with $v$ ).

From expression (14) for the twist of each link in the mechanism, we can derive an expression for the total kinetic energy. The kinetic energy $\mathcal{K}_{i}$ of link $i$ then follows as

$$
\begin{aligned}
\mathcal{K}_{i} & =\frac{1}{2}\left(v_{0 b}^{b}+J_{i}(q) \dot{q}\right)^{\top} \operatorname{Ad}_{g_{i b}}^{\top} I_{i} \operatorname{Ad}_{g_{i b}}\left(v_{0 b}^{b}+J_{i}(q) \dot{q}\right) \\
& =\frac{1}{2}\left[\left(v_{0 b}^{b}\right)^{\top} \dot{q}^{\top}\right] M_{i}(q)\left[\begin{array}{c}
v_{0 b}^{b} \\
\dot{q}
\end{array}\right]
\end{aligned}
$$


with

$$
M_{i}(q):=\left[\begin{array}{cc}
\operatorname{Ad}_{g_{i b}}^{\top} I_{i} \operatorname{Ad}_{g_{i b}} & \operatorname{Ad}_{g_{i b}}^{\top} I_{i} \operatorname{Ad}_{g_{i b}} J_{i} \\
J_{i}^{\top} \operatorname{Ad}_{g_{i b}}^{\top} I_{i} \operatorname{Ad}_{g_{i b}} J_{i}^{\top} \operatorname{Ad}_{g_{i b}}^{\top} I_{i} \operatorname{Ad}_{g_{i b}} J_{i}
\end{array}\right]
$$

where $J_{i}(q)$ is the geometric Jacobian of link $i$. The total kinetic energy of the mechanism is given by the sum of the kinetic energies of the mechanism links and the non-inertial base, that is,

$$
\mathcal{K}(q, v)=\frac{1}{2} v^{\top} \underbrace{\left(\left[\begin{array}{rr}
I_{b} & 0 \\
0 & 0
\end{array}\right]+\sum_{i=1}^{n} M_{i}(q)\right)}_{\text {inertia matrix } M(q)} v
$$

with $M(q)$ the inertia matrix of the total system.

We see that from (17) we can reformulate the expression in Egeland and Pettersen [8] for the inertia matrix in (6) with

$$
P_{i}(q)=\left[\begin{array}{ll}
\operatorname{Ad}_{g_{i b}} & \operatorname{Ad}_{g_{i b}} J_{i}
\end{array}\right] \in \mathbb{R}^{6 \times(6+n)} .
$$

Similarly the Coriolis matrix can be found by (7) where $W_{i}$ is given by (13) and $P_{i}$ by (19). $C(q, \zeta)$ is thus also welldefined.

\subsection{The Boundedness Property}

The dynamics as presented in Schjølberg [7] and Section 3.1 do not satisfy Property 1 . Due to the singularity there exist isolated points in the configuration space where the inertia matrix is singular. Even though this is the most common formulation of vehicle-manipulator systems in the literature this fact is normally not addressed in Lyapunov stability proofs. The formulation in Egeland and Pettersen [8] and Section 3.2 is globally valid and the inertia matrix is bounded in the whole configuration space. For systems where the configuration of non-Euclidean joints needs to be included in the dynamics, there does not seem to be a straight forward way to include the transformation between the local and global state variables without introducing singularities to the formulation.

This is, however, possible with the formulation presented in Section 3.3 where the inertia matrix is bounded for the whole configuration space also for non-Eucliden joints with a Lie group topology, such as $S O(3)$ and $S E(3)$. These formulations allow us to use the matrix representation $g_{0 i} \in$ $S E(3)$ of the configuration space and the structure of the configuration manifold is thus maintained.

\subsection{The Skew-Symmetric Property}

Schjølberg [7] shows that for the formulation presented in Section 3.1 the skew-symmetric property holds in bodyfixed coordinates. Based on this proof we can show that this property also holds for the approaches presented in Sections
3.2 and 3.3. First, for the Coriolis matrix given in (7) we can write

$$
\begin{aligned}
(\dot{M}-2 C)= & \frac{\mathrm{d}}{\mathrm{d} t}\left(\sum_{i=b}^{n} P_{i}^{\top}(q) I_{i} P_{i}(q)\right) \\
& -2 \sum_{i=b}^{n}\left(P_{i}^{\top}(q) I_{i} \dot{P}_{i}(q)-P_{i}^{\top}(q) W_{i} P_{i}(q)\right) \\
= & 2 \sum_{i=b}^{n} P_{i}^{\top}(q) W_{i} P_{i}(q)
\end{aligned}
$$

and $(\dot{M}-2 C)$ is skew-symmetric, for skew-symmetric $W_{i}$. Thus, the formulations given in Sections 3.1, 3.2 and 3.3 all satisfy the skew-symmetric property.

\section{Conclusions}

The boundedness property of the inertia matrix and the skew-symmetric property of the Coriolis matrix both depend on the choice of mathematical representation. The proofs of such properties thus need to be based on the particular representation chosen. In other words, a reference to a proof for a different choice of state variables or a different parameterisation of the matrices is not valid. In this paper we have shown that several widely used formulations of vehiclemanipulator dynamics do not possess these properties. We have also shown that some of the most commonly used references used for example in stability proofs of Lyapunov-based control laws in fact do not show these properties. As a result, many of the control laws presented in the literature are not valid.

For several formulations of vehicle-manipulator dynamics commonly found in the literature we have studied whether the boundedness and skew-symmetric properties hold. When we find the dynamic equations to satisfy these properties we have also included the proofs for future reference. These proofs have not previously been presented correctly for vehicle-manipulator systems. Finally we propose a modified version of the dynamic equations that satisfy both properties for general multibody systems.

\section{References}

[1] Antonelli, G., 2006. Underwater robots. Motion and force control of vehicle-manipulator systems. SpringerVerlag.

[2] Fossen, T. I., and Fjellstad, O. E., 1995. "Nonlinear modelling of marine vehicles in 6 degrees of freedom". International Journal of Mathematical Modelling Systems, 1, no. 1, pp. 17-28.

[3] Canudas de Wit, C., Diaz, E., and Perrier, M., 1998. "Robust Nonlinear Control of an Underwater Vehicle/Manipulator System with Composite Dynamics". In IEEE International conference on robotics and automation, Institute of electrical engineering INC (IEEE), pp. 452-457. 
[4] Schjolberg, I., and Fossen, T. I., 1994. "Modelling and control of underwater vehicle-manipulator systems". Proceedings of the 3rd Conference on Marine Craft Maneuvering and Control, Southampton, UK, pp. 4557.

[5] Murray, R. M., Li, Z., and Sastry, S. S., 1994. A Mathematical Introduction to Robotic Manipulation. CRC Press.

[6] Sciavicco, L., and Siciliano, B., 2005. Modelling and Control of Robot Manipulators. Springer.

[7] Schjolberg, I., 1996. "Modeling and control of underwater robotic systems". PhD thesis, Department of Engineering Cybernetics, Norwegian University of Science and Technology, Trondheim, Norway.

[8] Egeland, O., and Pettersen, K. Y., 1998. "Free-floating robotic systems". Control Problems in Robotics and Automation, 230, pp. 119-134.

[9] Borhaug, E., 2008. "Nonlinear control and synchronization of mechanical systems". PhD thesis, Department of Engineering Cybernetics, Norwegian University of Science and Technology, Trondheim, Norway.

[10] Ghorbel, F., Srinivasan, B., and Spong, M. W., 1998. "On the uniform boundedness of the inertia matrix of serial robot manipulators". Journal of Robotic Systems, 15, no. 1.

[11] Fossen, T. I., 2011. Handbook of Marine Craft Hydrodynamics and Motion Control. John Wiley \& Sons Ltd.

[12] Duindam, V., and Stramigioli, S., 2008. "Singularityfree dynamic equations of open-chain mechanisms with general holonomic and nonholonomic joints". IEEE Transactions on Robotics, 24(3), June, pp. 517-526.

[13] From, P. J., Duindam, V., Gravdahl, J. T., and Sastry, S., 2009. "Modeling and motion planning for mechanisms on a non-inertial base". In the Proceedings of International Conference of Robotics and Automation, Kobe, Japan. 